\title{
The PSI3 Agent Recommender System
}

\author{
Jorge J. Gómez-Sanz ${ }^{1}$, Juan Pavón ${ }^{1}$, and Áureo Díaz-Carrasco ${ }^{2}$ \\ ${ }^{1}$ Dep. Sistemas Informáticos y Programación, \\ Univ. Complutense, 28040 Madrid, Spain \\ \{jjgomez, jpavon\}@sip.ucm.es \\ http://grasia.fdi.ucm.es \\ 2 Ibermatica, \\ Avda. del Partenón, $16-18,1^{\circ}$ \\ 28042 Madrid, Spain \\ adiazcareibermatica.es \\ http://www.ibermatica.es
}

\begin{abstract}
This paper presents a multi-agent system (MAS) that implements a recommender system, essentially using collaborative filtering techniques. The design of the MAS is flexible to support the implementation of different filtering strategies and to control the global behavior of the system and its users. It has been applied in the PSI3 project to implement a personalized information dissemination service. Personalization requires some feedback from users in order to obtain some rating of their satisfaction with the information they receive. But, in order to make this effective, it has to be done with minimum disturbance to them. One way to achieve such purpose is by associating a personal agent for each user (to build and manage the user's profile) and a community agent for each group of users (to manage the dissemination and evaluation of information in the group, improve system performance, and prevent malicious behavior).
\end{abstract}

\section{Introduction}

The context of this work is the European project PSI3 (Personalized Services for Integrated Internet Information, www.psi3.org), whose main purpose was the provision of a set of tools for personalization of web sites. An important feature for personalization of a web site is the capability to suggest information of interest to users, based on their preferences (this is also known as recommender systems [1]). Basically, the problem consists on filtering from a set of elements those which may be of interest to the user, taking into account the matching of attributes of the user and the type of information (content-based filtering) or the experience from other users with similar profile (collaborative filtering [2]).

Collaborative filtering implies the classification and combination of preferences and opinions from users to determine groups of users with certain similarity. Then, the information of interest for a specific user can be derived from what is well rated in the groups to which the user is associated. The difference with content based filtering is that the answer of the system is determined by the behavior of the group, instead of a simple matching for a user profile with item attributes. Collaborative filtering is 
adequate, for instance, to recommend products such as books, movies, or web pages, where there is a significant degree of subjectivity in the user's choice.

There are already several agent-based systems implementing some collaborative filtering algorithms (for instance, [3] or [4]). Although they solve satisfactorily some specific problems, the architecture of these agent systems is not very flexible as all agents have strong dependencies between them in order to implement some concrete algorithm. The multi-agent system (MAS) presented in this paper intends to offer a more flexible architecture that would allow the implementation of different collaborative filtering algorithms, and even the possibility to combine these with content-based mechanisms, as it has been suggested in [5].

In this work, the collaborative filtering tool is conceived as a MAS, whose purpose is to provide users of a web community with personalized information. As such, the system needs to obtain some rating of the information presented to the users, but with minimum disturbance to them. This is achieved by associating a personal agent for each user, to build and manage the user's profile, and a community agent for each group of users, to manage the dissemination and evaluation of information in the group.

The interest of the proposed approach with respect to others is that this MAS is defined in terms of an organization and the associated workflows. These describe the collaborative filtering process and can be configured in different ways, not only by assigning values to certain parameters, but also by introducing social rules that determine the behavior of the group. This clarifies the design of the system since in the definition of the workflow we use simple terms and concepts closer to the intuitive definition of a process. It also provides flexibility because the workflow can be configured to behave in different ways, according to the kind of results that system administrators expect. Finally, this workflow can evolve. Actors in this workflow may change along the time. Therefore the behavior of the system can change too, but we can control its evolution to maintain some basic features. For instance, we can prevent from malicious behavior that could be (intentionally or not) introduced in the system by certain users (e.g., users doing spam or similar). Another characteristic of this system is the support for scalability, as the MAS is a distributed solution, which can support an arbitrary number of users. This is achieved by allowing system administrator to add new agent nodes for load sharing MAS components.

The organization of the MAS and its agents is described in section 2. There is special emphasis on the description of the main workflow in the organization and the parameters for its configuration, as the basis to structure the collaborative filtering algorithm. The complete design of the system, using the INGENIAS methodology [6], is available at http://grasia.fdi.ucm.es/ingenias/ejemplos/.

Section 3 discusses the definition of social rules to manage the behavior of a community. These can be used, for instance, to monitor the participation of users in the information filtering process and support the evolution of the community to some specific model, in which all the community users behave following certain patterns.

This system has been tested at two web sites (more information at www.psi3.org). Section 4 presents some results from this experimentation, which show that the application of the social rules described in section 3 contributes to reduce the number of interactions (i.e., improves system performance) and facilitate the evolution of the community of users to the desired configuration in a reasonable time. 
Based on the results of this work, the conclusions, in section 5, argue that the application of agent concepts facilitates the development, monitoring and evolution of recommender systems. These concepts, such as organization, social rules, workflows, goals, interactions, etc., can be used both at system level (e.g., configuration of workflows) and locally (e.g., personalization of the behavior of each agent). These ideas can be also applied to the design of other distributed applications that interact with a diverse and changing environment, involving different types of users.

\section{Organization and Workflows in the Design of the MAS}

The organization of the MAS is derived from the main workflow of the recommender system. Users with common interests are grouped in communities. Members of a community provide information to other members, who may find this information interesting or not. This information provision process can be understood as a document dissemination workflow. Participants in this workflow will determine agents in the system, their role (functionality), and interactions.

Users participate in the workflow through personal agents, one per user. Personal agents keep record of the experience of the user and some other attributes, and may act (with autonomy) on behalf of the user, thus minimizing user interactions with the system (for instance, in the process of rating the adequacy of a certain document, the personal agent can make the evaluation of the document). This means that the system can work whether the user is present or not. On the other hand, community agents act as group representatives, aim at reducing the number of interactions required between personal agents, and define policies for information dissemination and community membership. Community Agents are responsible of managing the information flow among Personal Agents.

There are several ways to improve the performance of the recommender system. First of all, by reducing the dissemination of irrelevant documents. This requires the participation of a subset of the members of the community in an evaluation process. If the evaluation is successful, then the document can be distributed to the community. In order to avoid too much involvement of users in this process (which could be annoying for them), personal agents can do such work. Personal agents build a user profile by taking into account those documents that were annotated positively by the user in the past. Example of annotations are: the user visited the document twice, the user recommended the document to other communities, or the user rated the document as good. Then, personal agents can check the adequacy of suggested documents against those of the user profile by means of text mining tools. Similarly, community agents can also check the adequacy of a document to the community.

This workflow, with the participation of agents, is shown in Fig. 1. It starts with a user or an agent, providing a document to the user's community. This document is checked against the profile of the community and if it passes the test, it is evaluated by a subset of the members of the community. If members evaluate it positively, the community disseminates the document to the rest of the members. The opinion of the members is an additional feedback for the document evaluation. Along the way, the document is compared against a user profile or the community profile, which are expressed here as a set of documents, using automated analysis (text mining tools 
such as Rainbow [7] in our case). This comparison occurs when the community receives the document and when an evaluator or a suggestion receiver receives the document.

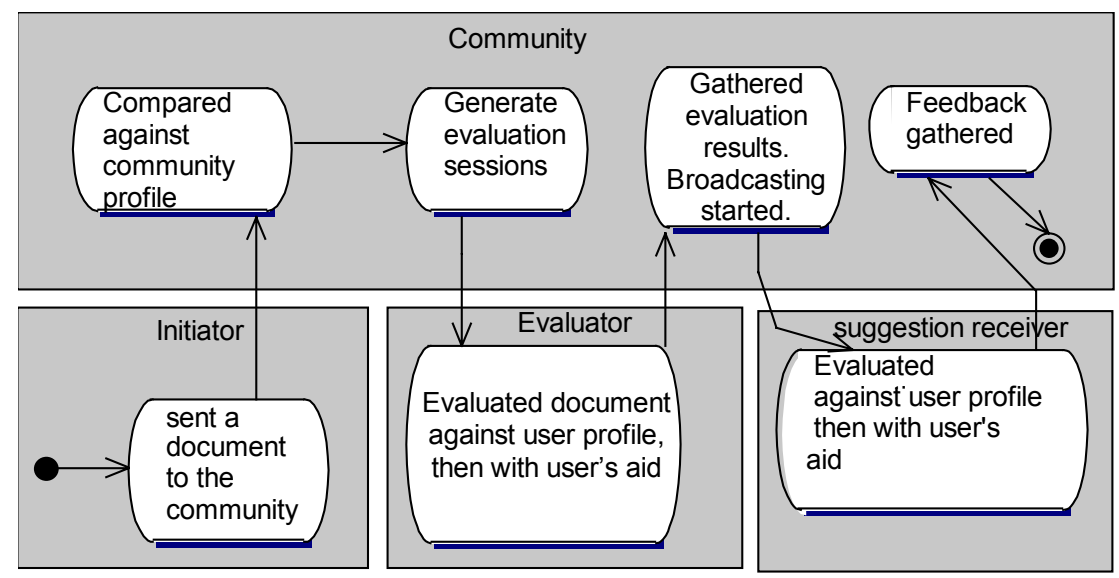

Fig. 1. Workflow implemented by agents to support collaborative filtering. Four actors execute the workflow: the initiator of the workflow, the community, the evaluator, and the suggestion receiver.

The workflow identifies the activities in the collaborative filtering process and the roles, which are responsible for them. Agents play roles, so they have the responsibility for performing the tasks that are assigned to the corresponding roles. In this system, personal agents undertake the roles of Initiator, Evaluator and Suggestion Receiver, whilst community agents undertake the role of Community. In this way, the identification of activities in the workflow and their assignment to roles is useful for defining which tasks and goals are assigned to each agent in the system.

The workflow can be configured at specific points with parameters (1), algorithms (2), and some rules (3 and 4):

1. The number of members who evaluate the information. The quality of the document depends highly on the number of users that have reviewed it.

2. Decision criteria for the autonomous evaluation of documents. These criteria determine whether the document fits into a profile (from the community or the user). A profile is initially defined as a set of positively evaluated documents. The default criterion is to consider the profile as a category and use a $k$-nearest neighbor algorithm with key word extraction [7] to obtain a degree of relevance to the profile. This degree has to be higher than a configurable threshold.

3. Decision criteria to consider evaluation results. Once users have evaluated documents, these criteria are used to decide whether or not dissemination should progress. The default criterion is to check if the number of positive evaluations is greater than the number of negative evaluations.

4. Criteria to act when a user ignores the document. Default behavior is to consider that the user is not interested in the document.

These workflow configuration features are those considered for the system that has been implemented in the PSI3 project and others could be considered. Each feature 
influences the behavior of the communities of users in which the workflow is executed. Users participate in the information flow because they benefit from the information received. If they do not feel satisfied with the documents they receive, then users will leave the community. However, there are many subtle differences in the attitude of users. There are users who do not feel confident enough to share their information with other users, but may collaborate in the information evaluation. Others just want to receive information and not collaborate at all. Also, there are users who only wish to disseminate information that perhaps does not interest other users, advertisements of products or services, for example. To take into account such attitudes, we have studied what types of communities may be interesting in a recommender system and how to control their evolution towards some of these types.

\section{Social Rules for Community Modeling}

A community can be described in terms of the types of users that constitute it. Type of user is defined in terms of the behavior in the information dissemination process, such as the following:

- An annoying user is one whose suggestions are not accepted by the majority of users.

- A passive user is one that does not review nor evaluate any document, but reads the supplied documents.

- An active user is one that participates in the evaluation workflow. It may be:

- An active reviewer, one that provides an evaluation on a document.

- An active suggestor, one that frequently provides documents to the community.

In order to improve the efficiency of the system, some types of users, e.g., annoying users, should be discouraged to participate in the community. Depending on the strategy of the recommender system, the ideal community can be defined as composed of users of specific types, for instance:

- A community where all users are active suggestors or active reviewers. The percentage of active reviewers should be higher than active suggestors so that reviewers are not overloaded by the amount of information to process.

- A community where all users are active suggestors, active reviewers or passive users. Ideally, there should be much more active reviewers than passive users. When there are more passive users than active reviewers and the number of reviewers is low, it is difficult to ensure document quality without overloading the reviewers.

Initially the system starts with any kind of community, as users subscribe to the community. By applying some social rules it is possible to evolve the community towards an ideal composition. A way to achieve this goal is with a rejection mechanism based on accumulated negative votes. The more negative votes a user provides, the more he is eligible for rejection. This policy is implemented in the form of rules managed by Community Agents. These rules are called social rules because their purpose is to tune the behavior of the community of agents attending to the social behavior of each one. Examples of such rules are: 
1. When the user does not review a document after a certain time, it means that there is a negative vote. Such users may be passive users. The interpretation is that the system punishes the passive user increasing the chance of rejection for that user.

2. When a user provides a negative evaluation for a document that is finally accepted by the community, it means that the user may be showing prejudices. Prejudices are punished with the assignment of negative votes to the user.

3. When a user suggests documents that are being badly evaluated, that means that there is a possible annoying user. In this situation, negative votes are generated.

The policy can be customized by system administrators to determine how many negative votes are needed to reject users, to ignore suggestions from annoying users or what is the punishment for passive users. Also, the number of rules can be increased at any time. So, system administrators can define new rules to deal with specific situations.

In this case, there is no rule that decreases the amount of negative votes (i.e., a kind of positive vote). Therefore, the system may end up rejecting members of the community that provide good information and with a very active participation in the community. To deal with this situation, personal agents can be programmed to subscribe again to the community that rejected them. This can be done transparently to the user. The community agent, instead of admitting them directly, inspects new subscribers to check if their profiles match those of the existing members of the community. Matching is performed with text mining tools [7]. This ensures that the community will accept users who provided good documents in the past.

The mechanism for the selection of reviewers is also important to determine the evolution of the community:

- Selecting the same person (for instance a community administrator) as a reviewer makes the community become a tyranny (a government in which a single ruler is invested with absolute power). This is usually the situation in moderated distribution lists. Depending on the skills of the moderator, this procedure may or may not work well. If the subject of the community is politics, the actuation of the moderator will undoubtedly influence the kind of documents that will be distributed. Also, the moderator becomes a bottleneck in the flow of information inside a community.

- $\quad$ Selecting among a fixed sub-set of users, who may be experts in the field, leads to oligarchy (government by a small group). This situation is desirable as long as selected users perform well.

- Voting who will act as reviewer leads to a democracy (government by the people, exercised either directly or through elected representatives). The reviewer role should be temporal and community members should present themselves as candidates for reviewing. Without a reward policy, we do not expect such collaboration from the members of a community. Another issue is that this solution overloads the users of the community.

The selection policy must ensure equality in information overload and provide participative evaluation criteria. This can be achieved by selecting a random set of users to act as reviewers. The selection process ensures that all users participate in a similar number of evaluation processes. This policy has been implemented in the PSI3 system, and the experimentation shows that with this simple policy the overload is low while the evaluation ratio can be kept high. 


\section{Testing PSI3 Agent Recommender System}

The final implementation of the recommender system in the PSI3 project, following the strategy discussed in the previous section, demonstrates that the policy of random selection of reviewers leads to low information overload and that the punishment policy leads to ideal communities for information dissemination.

To simulate exhaustive work of the system, the experiments run simulations of users accessing the PSI3 system. Each user is simulated by an agent whose profile is defined by a list of documents from the Reuters- 21578 collection. These documents, which are marked with tags, represent the preferences of the simulated user and are used to simulate human decision criteria (I like this document if its topics match those of my profile). The generation of the profile is done by selecting a distribution of documents (see table 1) according to a predefined set of categories. Each category corresponds to a topic keyword from the Reuters collection.

Table 1. Categories of users. The column on the left is the percentage of users that belong to each category. The top row indicates the categories of documents.

\begin{tabular}{|c|c|c|c|c|c|}
\hline Percentage/Categories & Soy-bean & Housing & Money-supply & Corn & Wool \\
\hline 25 & 0 & 0 & 0 & 100 & 0 \\
\hline 50 & 50 & 0 & 50 & 0 & 50 \\
\hline 25 & 0 & 0 & 100 & 50 & 0 \\
\hline
\end{tabular}

Initially the community under test is formed by $25 \%$ of users whose profile consists $100 \%$ of documents talking about corn (i.e., they are only interested on documents from this category), $50 \%$ whose profile consists of $50 \%$ of documents about soy-bean and 50\% about money-supply, and 25\% who are interested only in money-supply documents.

The tests start with a community composed of $30 \%$ of active suggestors (suggests, reviews and evaluates), $30 \%$ of active reviewers (reviews and evaluates) and $20 \%$ of passive users. The community uses 7 members to evaluate documents. These members are randomly selected.

The purpose of this experiment is to check whether the composition of the community evolves to a community where there are only active users who are interested in the topics of the community. The behavior of this community along the time is described in Fig. 2.

As it was expected the number of passive users, as well as the number of active users (suggestors and reviewers) that do not share common interests, decreases with time. Note that the number of active reviewers is related with the number of active suggestors. When the first increases, so does the second. Also, passive users influence negatively in the number of active suggestors. When the number of passive users increases, the number of active suggestors decreases. This means that in the workflow it is important to reject as soon as possible passive users so that active reviewers can support active suggestors with positive votes. This can achieved by modifying the number of negative votes that are needed to reject a user. 


\section{- - $\square$ - - Active reviewers $\longrightarrow$ - Active suggestors $\longrightarrow$ - Passive users}

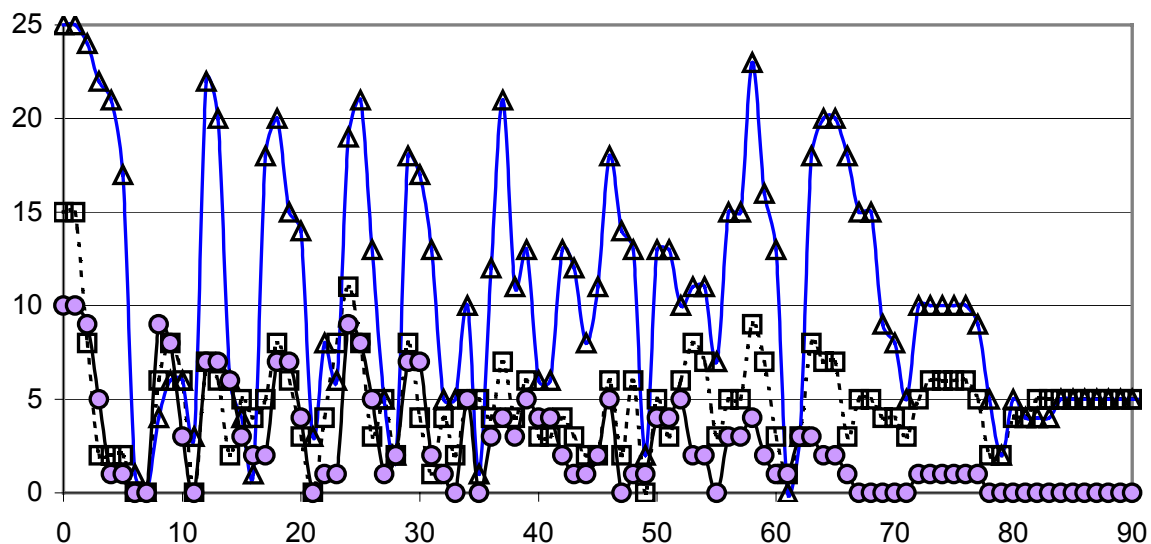

Fig. 2. Evolution of active users and passive users in the experiment. The $\mathrm{X}$-axis represents time in minutes and the $\mathrm{Y}$-axis represents the number of users in the experiment.

The system for the experiments was a Pentium III at $500 \mathrm{MHz}$ and $300 \mathrm{MB}$, running Linux. Agents were programmed with Java and the JESS rule engine system, which has considerable impact on performance. With this configuration, it took seventy minutes for the system to become stable with no passive users and ten active users. To achieve this state, the majority of the members in the community should evaluate some documents positively. These documents can be taken to build the community profile and are used to evaluate new candidates. This information is used by the community agent to determine whether new subscription requests may be accepted or not taking into account their potential to contribute with relevant documents to the community.

Another issue is how to measure the performance of the workflow. Traditional measurement in information retrieval establishes a widely accepted method (recall and precision). However, in real systems, such a measurement would not work because the user may simply have forgotten to review a piece of information that according to the system is highly rated. That is why in this case we preferred to collect how many times:

- A document was recognized as good and the user recognized it as good too.

- A document was recognized as good but the user did not think so.

- A document was recognized as good but the user did not review it.

On the other hand, it is also interesting to measure how good community agents are acting as filters. Again, similar measurements are applied:

- How many users have rated a piece of information (any) as good.

- How many users have rated a piece of information (any) as bad.

- How many users have rated a piece of information as good after the evaluation.

- How many users have rated a piece of information as bad after the evaluation.

- How many users have not reviewed the information. 
The information gathered by the system about these points appears in Fig. 3. As it was expected the ratio of users that received a suggested document is drastically inferior to the original. Having received 1400 suggestions from users of the community, these enable less than 400 high quality suggestions to be disseminated to the rest of the users. The documents that matched the categories associated to simulated users were more than 1100; meanwhile the documents rated negatively by simulated users were less than 600 . If all of the initial suggestions were accepted, then users may receive $1400 * 50$ documents what would not probably be well considered. Also, there is the problem of bandwidth. With this solution, bandwidth cost is reduced and users do not receive spam.

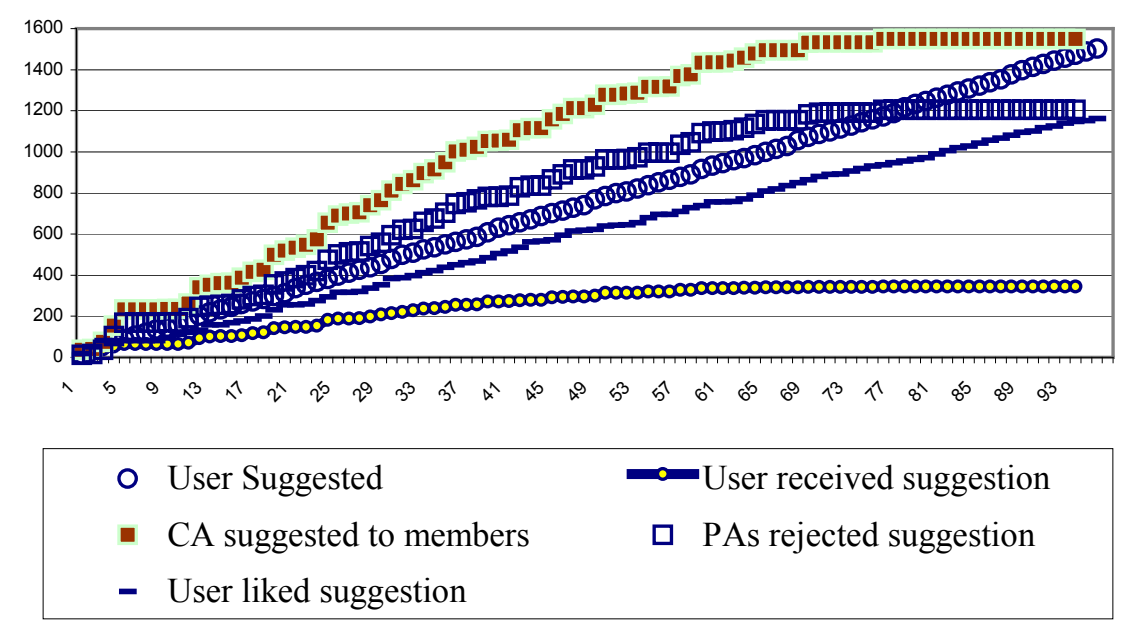

Fig. 3. Statistics on the performance of the workflow. These data represent accumulated values at a specific moment. The $\mathrm{X}$-axis represents time in minutes and the $\mathrm{Y}$-axis represents the accumulated number of times an event occurred.

\section{Conclusions}

The design of an agent-based recommender system around the specification of its workflows provides great flexibility. This is because the behavior of the MAS can be configured at different points in the workflow with parameters, algorithms, or rules. This enables experimentation until the desired behavior of a community of users is achieved. As an example, this paper shows the use of social rules to evolve a community of several types of users into a community of active users.

Social rules can be used to regulate the flow of information and community membership in the recommender system. This provides flexible ways to control some undesirable behavior by some users, for instance, those who insert advertising as interesting information. Social rules can specify how to detect people who annotate documents negatively too many times or who supply documents that are not 
considered as interesting by other members, and take the appropriate actions with them (for instance, ignore them or reject from the community).

User modeling can be quite simple, as in this case, where user profile consists of a collection of documents that were positively evaluated by the user. As already mentioned, this has the advantage of allowing the use of text mining tools for matchmaking agents.

Although current tests do not ensure that users will accept the results from this system better than others, experiments show that communities evolve to a number of users who are satisfied with the results, because the system promote users to participate proactively in the evolution of the community composition and improves the quality of the information received. We have focused on an evolution of the communities to relatively small communities where users are satisfied with the results they obtained from them, but other behaviors could be developed taking into account different strategies for recommender systems.

Acknowledgment. This work has been supported by the projects PSI3, a shared-cost RTD project under the 5th Framework Program of the European Commission (IST1999-11056), and INGENIAS, a project funded by Spanish Ministry of Science and Technology (TIC2002-04516-C03-03).

\section{References}

1. Resnick, P. and Varian, H. R.: Recommender systems. Communications of the ACM, vol. 40 , no. 3 (1997) 56-58.

2. Goldberg, D., Nichols, D., Oki, B. M., and Terry, D.: Using collaborative filtering to weave an information tapestry. Communications of the ACM, vol. 35, no. 12 (1992) 6170.

3. Delgado, J. and Ishii, N.: Multi-Agent Learning in Recommender Systems for Information Filtering on the Internet. International Journal of Cooperative Information Systems, vol. 10 , nos. 1\&2 (2001) 81-100.

4. Lashkari, Y., Metral, M., and Maes, P.: Collaborative Interface Agents. In Proc. Twelfth National Conference on Artificial Intelligence. AAAI Press (1994).

5. Schein, A., Popescul, A., Ungar, L., and Pennock, D.: Methods and Metrics for Cold-Start Recommendations. To appear in Proceedings of the 25'th Annual International ACM SIGIR Conference on Research and Development in Information Retrieval (SIGIR 2002).

6. Gómez-Sanz, J., Pavón, J.: Meta-modelling in Agent Oriented Software Engineering. In Proc. 8th Ibero-American Conference on AI (Iberamia 2002), LNAI 2527, Springer Verlag (2002) 606-615.

7. Andrew McCallum's: Bow: A Toolkit for Statistical Language Modeling, Text Retrieval, Classification and Clustering. http://www-2.cs.cmu.edu/ mccallum/bow/ 